\title{
Advanced Imaging Methods for Long-Baseline Optical Interferometry
}

\author{
Guy Le Besnerais, Sylvestre Lacour, Laurent M. Mugnier, Eric Thiébaut, Guy Perrin, and Serge Meimon
}

\begin{abstract}
We address the data processing methods needed for imaging with a long baseline optical interferometer. We first describe parametric reconstruction approaches and adopt a general formulation of nonparametric image reconstruction as the solution of a constrained optimization problem. Within this framework, we present two recent reconstruction methods, MIRA and WISARD, representative of the two generic approaches for dealing with the missing phase information. MIRA is based on an implicit approach and a direct optimization of a Bayesian criterion while WISARD adopts a self-calibration approach and an alternate minimization scheme inspired from radio-astronomy. Both methods can handle various regularization criteria. We review commonly used regularization terms and introduce an original quadratic regularization called "soft support constraint" that favors the object compactness. It yields images of quality comparable to nonquadratic regularizations on the synthetic data we have processed. We then perform image reconstructions, both parametric and nonparametric, on astronomical data from the IOTA interferometer, and discuss the respective roles of parametric and nonparametric approaches for optical interferometric imaging.
\end{abstract}

Index Terms-Fourier synthesis, image reconstruction, optical interferometry, phase closure.

\section{INTRODUCTION}

$\mathbf{T}$ HE ultimate resolution of an individual telescope is limited by its diameter. Because of size and mass constraints, today's technology limits diameters to $10 \mathrm{~m}$ or so for ground based telescopes and to a few meters for space telescopes. Optical interferometry (OI) allows one to surpass the resulting resolution limitation, currently by a few factors of ten, and in the next decade by a factor 100 .

Interferometers have allowed breakthroughs in stellar physics with the first measurements of diameter and more generally of fundamental stellar parameters, see recent reviews [1], [2]. Star pulsations have been detected allowing to understand both the physics of stars and the way they release matter in the interstellar medium. Also, the measurement of the pulsation of Cepheid

Manuscript received February 01, 2008; revised August 06, 2008. The associate editor coordinating the review of this manuscript and approving it for publication was Dr. Julian Christou.

G. Le Besnerais, L. M. Mugnier, and S. Meimon are with ONERA, 29, 92122 Châtillon Cedex, France (e-mail: lebesner@onera.fr; mugnier@onera.fr; meimon@onera.fr).

S. Lacour and G. Perrin are with the LESIA, Observatoire de Paris, 92190 Meudon, France (e-mail: sylvestre.lacour@obspm.fr; guy.perrin@obspm.fr).

E. Thiébaut is with CRAL, École Normale Supérieure de Lyon, 46, allée d'Italie, 69364 Lyon cedex 07, France (e-mail: thiebaut@obs.univ-lyon1.fr).

Color versions of one or more of the figures in this paper are available online at http://ieeexplore.ieee.org.

Digital Object Identifier 10.1109/JSTSP.2008.2005353 stars has allowed astronomers to establish an accurate distance scale in the universe. Many subjects have been addressed, a spectacular one being the shape of fast rotating stars, which are now clearly known to be oblate by sometimes a huge amount [3], [4]. With the advent of large telescopes and adaptive optics, more distant sources, beyond our own galaxy are now accessible. An important result has been the direct study of the dusty torus around super-massive black holes in the center of these galaxies, which is the corner stone of the unified theory to explain the active galactic nuclei phenomenon [5]-[7]. With the success of large interferometers, and especially of the European VLTI, interferometry is now used as a regular astrophysical tool by nonexpert astronomers and many more results are to be expected with the steadily increasing amount of published material.

OI consists in taking the electromagnetic fields received at each of the apertures of an array (elementary telescopes or mirror segments) and making them interfere. For each pair of apertures, the data contain high-resolution information at an angular spatial frequency proportional to the vector separating the apertures projected onto the plane of the sky, or baseline. With baselines of several hundred meters, this spatial frequency can be much larger than the cut-off frequency of the individual apertures.

Long baseline interferometers, for which the baseline-to-aperture ratio is quite large, usually provide a discrete set of spatial frequencies of the object brightness distribution, from which an image can be reconstructed by means of Fourier synthesis techniques. For the time being, interferometers able to provide direct images are not common: the Large Binocular Telescope (LBT), cf. 1bto . org/, will be the first of this kind with a baseline of the same order as the diameter of the two individual apertures. Recent, comprehensive reviews of OI and its history can be found for instance in [2], [8].

This paper addresses optical interferometry imaging (OII), i.e., the data processing methods needed for imaging sources with today's long baseline optical interferometers. Many reconstruction methods for OII are inspired from techniques developed for radio interferometry, as can be seen in the methods which were compared in the recent Interferometry Imaging Beauty Contests: IBC'04 [9] and IBC'06 [10]. Another body of work is the set of parametric reconstruction (a.k.a. model-fitting) methods. This latter class of methods is bound to remain a reference, partly because in interferometry, optical data will long remain much more sparse than radio data. In some instances, e.g. with the very extended object of IBC'06 [10], OII is very difficult even with relatively large data set, and thus often relies on the information provided by a parametric 
reconstruction. The latter is used at least as a guidance for judging the (nonparametric) image reconstruction, and often as a constraint for the support of the observed object, although this process is not always explicit.

We adopt a general formulation of nonparametric image reconstruction as the solution of a constrained optimization problem. Within this framework, methods may differ by many aspects, notably: the approximation of the data statistics, the type of regularization, the optimization strategy and the explicit or implicit accounting of missing phase information.

We present two recent nonparametric reconstruction methods, representative of the two generic approaches for dealing with the missing phase information. These nonparametric reconstruction methods are evaluated on synthetic and on astronomical data. The synthetic data allow us to study the influence of several types of prior knowledge. In particular, we show that contrarily to what is generally believed, appropriate quadratic regularizations are able to perform frequency interpolation and are suitable for the problem at hand if the object is compact: we propose a separable quadratic regularization which favors the object compactness and yields images of quality comparable to nonquadratic regularizations.

On the astronomical data we demonstrate the operational imaging capabilities of these methods; for these data, which may be considered representative of today's optical long-baseline interferometers, we show that the parametric approach remains a choice of reference for OII. Finally, we discuss the possible associations of both kinds of reconstruction methods.

The paper is organized as follows: Section II presents the instrumental process so as to define the observation model. Section III adresses the two main categories of prior information used for the reconstruction of the observed astronomical object: parametric models on the one hand, and regularization terms for nonparametric reconstruction methods on the other hand. Section V presents results on real data. Discussion and concluding remarks are gathered in Section VI.

\section{ObSERVATION MOdel OF LONG-BASELINE OPTICAL INTERFEROMETRY}

Let us consider a monochromatic source of wavelength $\lambda$ with a limited angular extension. Its brightness distribution can then be represented by $x(\boldsymbol{\xi}), \boldsymbol{\xi}=[\xi, \zeta]^{\mathrm{T}} \in \Omega$ with $\Omega$ a small portion of the plane of the sky around the mean direction of observation.

An intuitive way of representing data formation in a long-baseline interferometer is Young's double hole experiment, in which the aperture of each telescope is modeled by a (small) hole letting through the light coming from an object located at a great distance [11], [12]. At each observation time $t$, each pair $(k, \ell)$ of telescopes yields a fringe pattern with a spatial frequency of $\boldsymbol{b}_{k, \ell}(t) / \lambda$, where the baseline $\boldsymbol{b}_{k, \ell}(t)$ is the vector linking telescopes $k$ and $\ell$ projected onto the plane normal to the mean direction of observation. The coherence of the electromagnetic fields at each aperture is measured by the visibility or contrast $a_{k \ell}^{\text {data }}(t)$ and the position of the fringes, which are often grouped together in a complex visibility $y_{k \ell}^{\mathrm{data}}(t)=a_{k \ell}^{\mathrm{data}}(t) \exp \left(i \phi_{k \ell}^{\mathrm{data}}(t)\right)$. In an ideal experiment, the Van Cittert-Zernike theorem [11], [13] states that the coherence function (hence the complex visibility) is the Fourier transform (FT) of the flux-normalized object $\bar{x}=x / \int_{\Omega} x(\boldsymbol{\xi}) d \boldsymbol{\xi}$ at spatial frequency $\boldsymbol{\nu}=\boldsymbol{b}_{k, \ell}(t) / \lambda$. Let us introduce notations for Fourier quantities

$$
\begin{aligned}
y_{k \ell}(x, t) & =\int_{\boldsymbol{\xi} \in \Omega} \bar{x}(\boldsymbol{\xi}) \exp \left(-2 i \pi \boldsymbol{\xi}^{\mathbf{T}} \boldsymbol{b}_{k \ell} / \lambda\right) d \boldsymbol{\xi} \\
a_{k \ell}(x, t) & =\left|y_{k \ell}(x, t)\right| \\
\left.\phi_{k \ell}(x, t)\right) & =\arg \left\{y_{k \ell}(x, t)\right\} .
\end{aligned}
$$

In ground based interferometry, interferometric data are corrugated by the atmospheric turbulence. Inhomogeneities in the air temperature and humidity of the atmosphere generate inhomogeneities in the refractive index of the air, which perturb the propagation of light waves through the atmosphere. These perturbations lead to space and time variations of the input pupil phase $\varphi$, which can be modeled by a Gaussian spatio-temporal random process [14]-[16]. The spatial behavior of this process is generally described by the Fried's diameter $r_{0}$ [17]. The smaller $r_{0}$, the stronger the turbulence. Typically, its value is about $15-20 \mathrm{~cm}$ for $\lambda=0.55 \mu \mathrm{m}$ at good sites. The typical evolution time $\tau_{0}$ of the turbulent phase is given by the ratio $\tau_{0}$ of $r_{0}$ to the velocity dispersion of turbulence layers in the atmosphere $\Delta v$ [14]: a typical value is a few milliseconds at $\lambda=0.55 \mu \mathrm{m}$. In the sequel, short exposure (respectively, long exposure) refers to data acquired with an integration time shorter (respectively, markedly longer) than $\tau_{0}$.

\section{A. Short Exposure System Response}

For apertures of diameter $D$ notably larger than $r_{0}$, the loss of coherence due to the turbulence perturbations reduces the visibility of the fringes. This can be counterbalanced if the wavefronts are corrected by adaptive optics [18] (AO), at a rate faster than $\tau_{0}$, before the beams are made to interfere. In the sequel, we assume that each aperture is indeed either small enough or corrected by AO. Note, however, that it is possible to operate in the multi-speckle mode [19].

In the Young's holes analogy mentioned above, the remaining effect of turbulence on interferometric measurements is to add a phase shift (or piston) $\varphi_{k}(t)$ at each aperture $k$ to the wave going through it. The interference between two apertures $k$ and $\ell$ are thus out of phase by a random "differential piston" $\varphi_{\ell}(t)-$ $\varphi_{k}(t)$, whose typical evolution time is of the order of $\tau_{0}$ and depends on the baseline [20].

A short exposure observation finally writes

$$
\begin{aligned}
a_{k \ell}^{\text {data }} & =a_{k \ell}(x, t)+\text { noise } \\
\phi_{k \ell}^{\text {data }}(t) & =\phi_{k \ell}(x, t)+\varphi_{\ell}(t)-\varphi_{k}(t)+\text { noise }[2 \pi] .
\end{aligned}
$$

When a complete interferometer array of $N_{\mathrm{T}}$ telescopes is used, i.e., one in which all the possible two-telescope baselines can be formed simultaneously, there are $N_{\mathrm{B}}=N_{\mathrm{T}}\left(N_{\mathrm{T}}-1\right) / 2$ visibility phase measurements (5) for each instant $t$. These equations can be put in matrix form

$$
\boldsymbol{\phi}^{\mathrm{data}}(t)=\boldsymbol{\phi}(\boldsymbol{x}, t)+\boldsymbol{B} \boldsymbol{\varphi}(t)+\text { noise }[2 \pi]
$$

where the baseline operator $B$ of dimensions $N_{\mathrm{B}} \times N_{\mathrm{T}}$ is formally defined in Appendix A. 




Fig. 1. Frequency coverages obtained with the IOTA interferometer on $\chi$ Cygni (observing run of May 2006). For a given baseline $\boldsymbol{b}_{k, \ell}(t)$ and a given spectral channel of mean wavelength $\lambda$, the measured frequency is $\boldsymbol{\nu}=[u, v]^{\mathrm{T}}=$ $\boldsymbol{b}_{k, \ell}(t) / \lambda$.

Note that the baseline $\boldsymbol{b}_{k, \ell}(t)$ between apertures $k$ and $\ell$ depends on time. Indeed, the aperture configuration as seen from the object changes as the Earth rotates. It is thus possible to use "Earth rotation synthesis", a technique that consists, when the source emission does not vary in time, in repeating the measurements in the course of a night of observation to increase the frequency coverage of the interferometer. A typical frequency coverage obtained with the IOTA interferometer (see Section V-A) is presented in Fig. 1. This Fourier coverage can be formally represented by a short-exposure transfer function

$$
\widehat{h}(\boldsymbol{\nu})=\sum_{t} \sum_{k, \ell} \eta_{k, \ell}(t) e^{i\left(\varphi_{\ell}(t)-\varphi_{k}(t)\right)} \delta\left(\boldsymbol{\nu}-\boldsymbol{b}_{k, \ell}(t) / \lambda\right)
$$

where $\delta$ denote the Dirac function and summations extend over all observation instants and used pairs of telescope.

The complex gains $\eta_{k, \ell}(t)$ account for visibility losses that originate from various causes. Some of them can be estimated using observations of a calibrator (i.e., a star unresolved by the interferometer, or whose diameter is precisely known, located near the object of interest and with similar spectral type) and compensated for. In the following, we consider that the $\eta_{k, \ell}(t)$ are pre-calibrated, i.e., $\eta_{k, \ell}(t)=1$.

Equation (7) and Fig. 1 provide a first insight on the data processing problem at hand. It is a Fourier synthesis problem, i.e., it consists in reconstructing an object from a sparse subset of its Fourier coefficients. As shown by Fig. 1, interferometry gives access to very high frequency coefficients, but the number of data is very limited (a few hundreds). Measuring these data with a sufficient signal-to-noise ratio (SNR) is quite delicate. Indeed, in a short exposure, the differential pistons are expressed by random displacements of the fringes without attenuation of the contrast. But in long exposure measurements, averaging these displacements leads to a dramatic visibility loss: a specific averaging process must be used, as described in the next section.

\section{B. Long Exposure Data}

1) Principle: As mentioned above, the main obstacle to long exposure data measurement is the differential pistons $\varphi_{\ell}(t)$ which affect the phase of the visibility. On the one hand, averaging the modulus of the visibility is possible; on the other hand, some phase information can be obtained by carrying out phase closure [21] before the averaging. The principle is to sum short-exposure visibility phase data $\phi_{k \ell}^{\text {data }}(t) \phi_{\ell m}^{\text {data }}(t)$ and $\phi_{m k}^{\text {data }}(t)$ measured on a triangle of telescopes $\left(T_{k}, T_{\ell}, T_{m}\right)$. From (5), one can check that turbulent pistons are canceled out in the closure phase defined by

$$
\begin{aligned}
\beta_{k \ell m}^{\mathrm{data}}(t) & \triangleq \phi_{k \ell}^{\mathrm{data}}(t)+\phi_{\ell m}^{\mathrm{data}}(t)+\phi_{m k}^{\mathrm{data}}(t)+\text { noise }[2 \pi] \\
& =\phi_{k \ell}(x, t)+\phi_{\ell m}(x, t)+\phi_{m k}(x, t)+\text { noise}[2 \pi] \\
& =\beta_{k \ell m}(x, t)+\text { noise }[2 \pi] .
\end{aligned}
$$

To form this type of expression it is necessary to measure three visibility phases simultaneously, and thus to use an array of three telescopes or more.

In the case of a complete interferometer array of $N_{\mathrm{T}}$ telescopes, the set of closure phases that can be formed is generated by, for instance, the $\beta_{1 k \ell}(t), 1<k<\ell \leq N_{\mathrm{T}}$, i.e., the closure phases measured on the triangles of telescopes including telescope $T_{1}$. There are $\left(N_{\mathrm{T}}-1\right)\left(N_{\mathrm{T}}-2\right) / 2$ of these independent closure phases. In what follows, the vector grouping together these independent closure phases will be noted $\beta^{\text {data }}$ and a closure operator $\boldsymbol{C}$ is defined such that

$$
\boldsymbol{\beta}^{\text {data }}(t) \triangleq \boldsymbol{C} \boldsymbol{\phi}^{\text {data }}(t)=\boldsymbol{C} \boldsymbol{\phi}(\boldsymbol{x}, t)+\text { noise }[2 \pi] .
$$

The second equation is a matrix version of (8): the closure operator cancels the differential pistons, a property that can be written $\boldsymbol{C B}=\mathbf{0}$, with $\boldsymbol{B}$ the baseline operator introduced in (6) and Appendix A. It can be shown [22] that this equation implies that the closure operator has a kernel of dimension $N_{\mathrm{T}}-1$, given by

$$
\operatorname{Ker} \boldsymbol{C}=\left\{\bar{B} \boldsymbol{\alpha}, \boldsymbol{\alpha} \in \mathbb{R}^{N_{\mathrm{T}}-1}\right\}
$$

where $\bar{B}$ is obtained by removing the first column from $\boldsymbol{B}$. The closure phase measurement thus does not contain all the phase information. This classical result can also be obtained by counting up the phase unknowns for each instant of measure $t$. There are $N_{\mathrm{T}}\left(N_{\mathrm{T}}-1\right) / 2$ unknown object visibility phases and $\left(N_{\mathrm{T}}-1\right)\left(N_{\mathrm{T}}-2\right) / 2$ independent measured phase closures, which gives $N_{\mathrm{T}}-1$ missing phase data. In other words, optical interferometry through turbulence is a Fourier synthesis problem with partial phase information. As is well known, the more apertures in the array, the smaller the proportion of missing phase information.

2) Data Reduction and Averaging: In practice, the basic observables of optical interferometry are then sets of three simultaneous fringe patterns obtained on a triangle of telescopes. The output of the pre-processing stage (see for instance [23] for a description of the pre-processing with IOTA data) are as follows.

- Power spectra $V_{k \ell}^{(2) \text {,data }}(t)$ :

$$
V_{k \ell}^{(2), \text { data }}(t)=\left\langle\left|y_{k \ell}^{\text {data }}(t+\tau)\right|^{2}\right\rangle_{\tau}
$$


- Bispectra $V_{j k \ell}^{(3), \text { data }}(t), j<k<\ell$, defined by

$$
\left\langle y_{j k}^{\mathrm{data}}(t+\tau) y_{k \ell}^{\mathrm{data}}(t+\tau) y_{l_{j}}^{\mathrm{data}}(t+\tau)\right\rangle_{\tau}
$$

Notation $\langle.\rangle_{\tau}$ expresses the averaging in a time interval around instant $t$. The integration time $\tau$ must be short enough for the spatial frequency to be considered constant during the integration despite the rotation of the Earth. It also impacts the standard deviation of the residual noises on the measurement. Equation (10) and (11) are biased estimates of the object spectrum and bispectrum, see [24], [25] for the expressions of these bias.

The phases of the bispectra

$$
\beta_{j k \ell}^{\mathrm{data}}(t) \triangleq \arg \left\{V_{j k \ell}^{(3), \text { data }}(t)\right\}, \quad j<k<\ell
$$

constitute unbiased long-exposure closure phase estimators.

3) Observation Model and Data Likelihood: Using notation $s_{k \ell}^{\text {data }}(t)=V_{k \ell}^{(2), \text { data }}(t)$ and concatenating all instantaneous measurements in vectors denoted by bold letters, the long-exposure observation model writes

$$
\left\{\begin{array}{l}
\boldsymbol{s}^{\text {data }}(t)=\boldsymbol{a}^{2}(x, t)+\boldsymbol{s}^{\text {noise }}(t) \\
\boldsymbol{\beta}^{\text {data }}(t)=\boldsymbol{C} \phi(x, t)+\boldsymbol{\beta}^{\text {noise }}(t)[2 \pi]
\end{array} .\right.
$$

Noise terms are usually only characterized by estimated secondorder statistics, hence they are modeled as Gaussian processes: $\boldsymbol{s}^{\text {noise }}(t) \sim \mathcal{N}\left(0, \boldsymbol{R}_{\boldsymbol{s}(t)}\right), \boldsymbol{\beta}^{\text {noise }}(t) \sim \mathcal{N}\left(0, \boldsymbol{R}_{\boldsymbol{\beta}(t)}\right)$. Covariance matrices $\boldsymbol{R}_{\boldsymbol{s}(t)}$ and $\boldsymbol{R}_{\boldsymbol{\beta}(t)}$ are generally assumed to be diagonal (as, for instance in the OIFITS data exchange format [26]), although correlations are for instance produced by the use of the same reference stars in the calibration process [27].

Note that the observation model (12) corresponds to a minimal dataset for a complete $N_{\mathrm{T}}$-telescope interferometer. In practice, the data may contain closures without the corresponding power spectra, or bispectra amplitudes. These supplementary data are not processed the same way by all the data reconstruction methods, in particular by the MIRA and WISARD algorithms described in Section IV.

The neg-log-likelihood derived from this observation model writes

$$
J^{\text {data }}(x)=\sum_{t}\left\{\chi_{\boldsymbol{s}}^{2}(x, t)+\chi_{\boldsymbol{\beta}}^{2}(x, t)\right\} .
$$

The notation $\chi_{\boldsymbol{s}}^{2}(x, t)$ denotes the $\chi^{2}$ statistics of the squared visibility residuals at time $t$

$\chi_{\boldsymbol{s}}^{2}(x, t)=\left(\boldsymbol{s}^{\mathrm{data}}(t)-\boldsymbol{a}^{2}(\boldsymbol{x}, t)\right)^{\mathrm{T}} \boldsymbol{R}_{\boldsymbol{s}(t)}^{-1}\left(\boldsymbol{s}^{\mathrm{data}}(t)-\boldsymbol{a}^{2}(\boldsymbol{x}, t)\right)$.

In the sequel, we shall use the term "likelihood" to denote the various goodness-to-fit terms such as (13) derived from the distribution of the data.

The closure term $\chi_{\boldsymbol{s}}^{2}(x, t)$ is usually also a $\chi^{2}$ over phase closures residuals, but in order to account for phase wrapping and to avoid excessive nonlinearity, the term $\chi_{\boldsymbol{\beta}}^{2}(x, t)$ related to the measured phase closures can also be chosen as a weighted quadratic distance between the complex phasors

$$
\chi_{\boldsymbol{\beta}}^{2}(x, t)=\sum_{j<k<\ell} \frac{1}{\operatorname{Var}\left[\beta_{j, k, \ell}^{\mathrm{data}}(t)\right]}\left|\mathrm{e}^{i \beta_{j, k, \ell}^{\mathrm{data}}(x, t)}-\mathrm{e}^{i \beta_{j, k, \ell}(x, t)}\right|^{2}
$$

where $\boldsymbol{\beta}(x, t)=\boldsymbol{C} \phi(x, t)$ is the model of the measured phase closure $\boldsymbol{\beta}^{\text {data }}(t)$.

\section{OBJECT MODELS}

Imaging amounts to finding a flux-normalized positive function $x$ defined over the support $\Omega$ which fits the data (12). One way is to minimize the likelihood (13). Three problems are then encountered.

1) Under-determination: because of the noise, the object which minimizes the likelihood is not necessarily the good solution: actually, several objects are compatible with the data. This is a usual situation in statistical estimation, which is here emphasized by the small number of measured Fourier coefficients, the noise level and the missing phase information.

2) Nonconvexity: the phase indetermination leads to a non convex ${ }^{1}$ and often multi-modal data likelihood.

3) Non-Gaussian likelihood: phase and modulus measurements with Gaussian noise leads to a non-Gaussian likelihood in $x$. In other words, even if all the visibility phases were measured instead of just the closure phases, the data likelihood would still be nonconvex. We shall come back to this point in Section IV-B.

To deal with under-determination, one is led to assume some further prior knowledge on the object. In this section we review two approaches: parametric modeling and regularized reconstruction.

\section{A. Parametric Models}

1) Introduction: The object is sought by minimization of (13) using a parametric form $x(\boldsymbol{\theta})$. The resulting criterion $\boldsymbol{\theta} \mapsto J^{\text {data }}(x(\boldsymbol{\theta}))$ often exhibits further nonlinearities, but as the number of parameters is very limited (typically $\operatorname{dim}(\boldsymbol{\theta}) \leq 5$ ) global minimization is achievable. The minimal value of the criterion gives an information on whether the chosen model is appropriate to describe the brightness distribution of the object. Additionally, the second derivative of $J^{\text {data }}(x(\boldsymbol{\theta}))$ around its minimum allows the estimation of error bars.

For years, interferometric data were very sparse, essentially because the number of telescopes in interferometers was quite small. Most interferometers were two-telescope arrays and in few cases three telescopes were available. The only way to interpret the data was then to use parametric models with a very small number of parameters, typically two or three. Among the most used models, let us mention the uniform disk to measure stellar diameters, and binary system models.

When objects are as simple as individual or binary regular stars, such simple models can be used beforehand to prepare the observations and anticipate likely visibility values. This is very useful to conduct "baseline bootstrapping", a process which consists in observing a visibility of very low SNR using a triangle of telescopes with two other baselines having a higher SNR. Simple parametric models are also used to compute the expected visibility of reference stars in order to calibrate the

${ }^{1}$ Convexity is a desirable property of a criterion when a minimization process is conducted, which can furnish sufficient conditions for convergence of iterative local optimization techniques toward a global minimum. A well-known reference is the book by R.T. Rockafellar [28]. 
response of the interferometer and overcome residual visibility losses, such as those due to polarization effects.

Now that current interferometers yield richer data, more sophisticated models can be used. It is outside the scope of this paper do describe the large number of parametric models which are used nowadays, however we present in the following subsections two trends in parametric modeling.

2) Fitting of a Geometrical Model: Parametric inversion can be used to derive the geometrical structure of the brightness distribution of the object. An example among others is the derivation of the brightness profile $I$ of a limb-darkened disk. Limb darkening is an optical depth effect, which results in a drop of the effective temperature (and hence intensity) towards the edge of the stellar disk. Numerous types of limb-darkening models exist in the literature. To cite only two, one can use a power law [29] as follows:

$$
I(\rho) / I(1)=\rho^{\alpha}
$$

or a quadratic law [30]

$$
I(\rho) / I(1)=1-a(1-\rho)-b(1-\rho)^{2}
$$

where $\rho$, the cosine of the azimuth of a surface element of the star, is equal to $\sqrt{1-\left(2 r / \theta_{\mathrm{LD}}\right)^{2}}, r$ being the angular distance from the star center, and $\theta_{\mathrm{LD}}$ is the angular diameter of the photosphere. The parametric fit is actually done on complex visibilities. In the Fourier domain, the power law limb darkening model yields [31]

$$
y\left(v_{r} ; \boldsymbol{\theta}\right)=\sum_{k \geq 0} \frac{\Gamma(\alpha / 2+2)}{\Gamma(\alpha / 2+k+2) \Gamma(k+1)}\left(\frac{-\left(\pi v_{r} \theta_{\mathrm{LD}}\right)^{2}}{4}\right)^{k}
$$

where the parameters are $\boldsymbol{\theta}=\left[\alpha, \theta_{\mathrm{LD}}\right]^{\mathrm{T}}, v_{r}$ is the radial spatial frequency and $\Gamma$ the Euler gamma function $(\Gamma(k+1)=k !)$.

The quadratic law model yields

$$
y\left(v_{r} ; \boldsymbol{\theta}\right)=\frac{(1-a-b) \frac{J_{1}(\zeta)}{\zeta}+\frac{a+2 b}{\sqrt{2 / \pi}} \frac{J_{3 / 2}(\zeta)}{\zeta^{3 / 2}}-2 b \frac{J_{2}(\zeta)}{\zeta^{2}}}{1 / 2-a / 6-b / 12}
$$

where the parameters are $\boldsymbol{\theta}=\left[a, b, \theta_{\mathrm{LD}}\right]^{\mathrm{T}}, \zeta=\pi v_{r} \theta_{\mathrm{LD}} ; J_{1}$ and $J_{2}$ are the first and second-order Bessel functions, respectively, and

$$
J_{3 / 2}(\zeta)=\sqrt{\frac{2}{\pi \zeta}}\left(\frac{\sin (\zeta)}{\zeta}-\cos (\zeta)\right)
$$

3) Physical Parameter Determination: An interesting possibility offered by parametric inversion is to directly adjust physical parameters of the objects. An example can be found on a study about the star $\mu$ Cep from FLUOR interferometric observation [32]. Data was fitted with an analytical expression of the brightness distribution that includes a temperature for the photosphere and a radiative transfer model of the molecular layer. The model used is radial, and writes

$x\left(r=\sqrt{p^{2}+q^{2}} ; \boldsymbol{\theta}\right)=B\left(\lambda, T_{\star}\right) \exp \left(\frac{-\tau(\lambda)}{\sqrt{1-r^{2} / \Theta_{\text {layer }}^{2}}}\right)$

$$
+B\left(\lambda, T_{\text {layer }}\right)\left[1-\exp \left(\frac{-\tau(\lambda)}{\sqrt{1-r^{2} / \Theta_{\text {layer }}^{2}}}\right)\right]
$$

for $\sin (\theta) \leq \Theta_{\star} / \Theta_{\text {layer }}$ and

$$
x(r ; \boldsymbol{\theta})=B\left(\lambda, T_{\text {layer }}\right)\left[1-\exp \left(\frac{-2 \tau(\lambda)}{\sqrt{1-r^{2} / \Theta_{\text {layer }}^{2}}}\right)\right]
$$

otherwise, where the parameters are $\boldsymbol{\theta}=\left[\Theta_{\star}, \Theta_{\text {layer }}, T_{\text {layer }}, \tau(\lambda)\right]$, with $\Theta_{\star}$ and $\Theta_{\text {layer }}$ the diameters of the star and the molecular layer respectively, and $\tau(\lambda)$ the opacity of the molecular layer as a function of the wavelength. $B\left(\lambda, T_{\text {layer }}\right)$ is the Planck function.

This model illustrates how to obtain a direct estimation of the temperatures of the star and of the molecular layer from interferometric data. Interestingly, this type of model allows an exploitation of multi-wavelength observations that takes into account the chromaticity of the astronomical object.

\section{B. Regularized Reconstruction}

1) Introduction: In this framework, the sought object distribution $x$ is represented by its projection onto a basis of functions, often defined as a shifted separable kernel basis

$$
x(\boldsymbol{\xi})=\sum_{p=0}^{P-1} \sum_{q=0}^{Q-1} x(p, q) \gamma(\xi-p \Delta \xi) \gamma(\zeta-q \Delta \zeta)
$$

where dimensions $P, Q$ and sampling steps $\Delta \xi, \Delta \zeta$ are chosen so as to span the object support $\Omega$ and to satisfy the Shannon-Nyquist condition with respect to the experimental frequency coverage. Kernels $\gamma$ are often box functions or sinc functions, sometimes wavelets or prolate spheroidal functions [33], [34]. The estimation aims at finding the coefficients $\boldsymbol{x}=[x(0,0), \ldots x(P-1, Q-1)]^{\mathrm{T}}$ so as to fit the data. This approach is sometimes loosely called a non parametric approach because the parametrization (22) is here not to put further constraints on the solution, but only to allow its numerical computation. To tackle the under-determination the data likelihood (13) is combined with a regularization term in a criterion of the form

$$
J(\boldsymbol{x})=J^{\text {data }}(\boldsymbol{x})+\mu J^{\text {prior }}(\boldsymbol{x}) .
$$

The regularization term $J^{\text {prior }}$ enforces the desired properties of the object (smoothness, positivity, compactness, etc.). The regularization parameter $\mu$ allows to tune the regularization strength or, equivalently, to select a data term level set $\left\{J^{\text {data }}(x)<\epsilon\right\}$. Of course, the choice of $\mu$ depends on the noise level. The compound criterion (23) can be derived within a Bayesian paradigm: the data model (12) is translated into a data likelihood (13) which is combined with a prior distribution on the object by Bayes' rule to form the a posteriori distribution. Maximization of the posterior distribution is equivalent to minimization of the likelihood, i.e., of a regularized criterion such as (23).

Most regularization terms penalize the discrepancy between the current solution and some a priori object $\boldsymbol{x}^{\text {prior }}$, be it simply the null object. In the OII context of very sparse and ambiguous 
datasets, the use of a meaningful prior object can be an efficient way to orient the reconstruction and to improve the results. In IBC'06 for instance, an a priori object was finally provided to the participants: see [10, Fig. 3]. $\boldsymbol{x}^{\text {prior }}$ can be obtained from previous observations of the source with other instruments, or derived from the fit of a parametric model to the interferometric data at hand-see Section V-C below.

In the following, we briefly discuss the choice of the regularization terms and introduce an original regularization criterion that can be used on compact objects to enforce a "soft support" constraint.

2) Quadratic Regularization: Quadratic regularization has been applied to Fourier synthesis and OII by A. Lannes et al. [34]-[36]. For relatively smooth objects, one can use a correlated quadratic criterion expressed in the Fourier domain, with a parametric model of the object's power spectrum $S_{x}$. Such a model was proposed for deconvolution of AO corrected images in [37]

$$
S_{x}(v=|\boldsymbol{\nu}|)=k /\left[v_{0}^{p}+v^{p}\right]-\left|\mathrm{FT}\left\{\boldsymbol{x}^{\text {prior }}\right\}(\boldsymbol{\nu})\right|^{2}
$$

This model, which relies on a prior object and three "hyper-parameters" $\left(k, v_{o}, p\right)$ has been used in various image reconstruction problems, including OII [9], [38]. Parameter $v_{0}$ is a cutoff frequency which is typically the inverse of the diameter of the object's support and avoids divergence at the origin, $p$ characterizes the decrease rate of the object's energy in the Fourier domain, and $k$ plays the role of the inverse of hyperparameter $\mu$ of (23) and can replace this parameter. As already mentioned, an advantage of quadratic criteria is that it is possible to estimate the hyper-parameters, by maximum likelihood for example [39].

A simple and efficient quadratic regularization is a separable quadratic distance to the prior object $\boldsymbol{x}^{\text {prior }}$. In Appendix B, we show that the general expression of such regularization terms under the OII-specific constraints of unit sum and positivity [see (40)] is

$$
J^{\text {prior }}(\boldsymbol{x})=\sum_{p, q} x(p, q)^{2} / x^{\text {prior }}(p, q)
$$

where the a priori object $\boldsymbol{x}^{\text {prior }}$ is chosen to be strictly positive and normalized to unity.

In the absence of a meaningful object to be used for $x^{\text {prior }}$, the width $w$ of the observed source is usually more or less known, so we have found that a reasonable a priori object is an isotropic one such as the Lorentzian model $x^{\text {prior }}(p, q) \propto 1 /\left(1+2\left(p^{2}+\right.\right.$ $\left.\left.q^{2}\right) / w^{2}\right)$. Such a prior object can then be seen as enforcing a loose support constraint.

3) Edge-Preserving Regularization: For extended objects with sharp edges such as planets, a quadratic criterion tends to over-smooth the edges and introduce spurious oscillations, or ringing, in their neighborhood. A solution is thus to use an edge-preserving criterion such as the so-called quadratic-linear, or $L_{2}-L_{1}$ criterion, which are quadratic for weak gradients of the object and linear for the stronger ones. The quadratic (or $L_{2}$ ) part ensures good noise smoothing and the linear (or $L_{1}$ ) part cancels edge penalization. Here we present an isotropic version [40] of the criterion proposed by Rey [41] in the context of robust estimation and used by Brette and Idier in image restoration [42]

$$
\begin{aligned}
J^{\text {prior }}(\boldsymbol{x}) & =\delta^{2} \sum_{p, q} \psi(g(p, q) /(s \delta)) \text { where } \\
g(p, q) & =\sqrt{\nabla_{\xi} \boldsymbol{x}(p, q)^{2}+\nabla_{\zeta} \boldsymbol{x}(p, q)^{2}} \\
\psi(z) & =z-\ln (1+z)
\end{aligned}
$$

with $\nabla_{\xi} \boldsymbol{x}$ and $\nabla_{\zeta} \boldsymbol{x}$ the gradient approximations by finite differences in the two spatial directions. The two parameters to be adjusted are a scale parameter $s$ and a threshold parameter $\delta$. Parameter $s$ plays the same role as the conventional regularization parameter $\mu$ and can replace it, with $1 / s^{2}=\mu$; indeed, for small values of $g(p, q)$ each term of (26) reads $\delta^{2} \psi(g(p, q) /(s \delta)) \simeq$ $1 /\left(2 s^{2}\right) \times g^{2}(p, q)$.

4) Spike-Preserving or Entropic Regularization: For objects composed of bright points on a fairly extended and smooth background, such as are often found in astronomy, a useful regularization tools is entropy. Here, we adopt the pragmatic point of view of Narayan and Nityananda [43] and consider that entropy is essentially a separable criterion

$$
J^{\text {prior }}(\boldsymbol{x})=\sum_{p, q} h\left(x(p, q), x^{\text {prior }}(p, q)\right)
$$

where each pixel is drawn toward a prior value $x^{\text {prior }}(p, q)$ according to a nonquadratic potential $h$ also termed neg-entropy. Classical examples of "entropic potential" are the Shannon entropy $h_{S}\left(x, x^{\text {prior }}\right)=x \log \left(x / x^{\text {prior }}\right)$ and the Burg entropy $h_{B}\left(x, x^{\text {prior }}\right)=-\log \left(x / x^{\text {prior }}\right)$ but many other non quadratic potentials can be used, as shown in [43]. The major interest of the nonlinearity of entropic potentials is that they help to interpolate holes in the frequency coverage. Side effects are emphasizing spikes and smoothing low level structures. As it result in ripples suppression in the flat background and enhanced spatial resolution near sharp structures, this behavior may be considered as beneficial in the context of interferometric imaging though it also introduces substantial biases. Note that the interferometric imaging method BSMEM [44], winner of the IBC's 2004 and 2006 [9], [10], or the VLBMEM method [9] are based upon entropic regularization with potential $h_{S}$.

Here we propose an entropic-like criterion which re-employs the potential $\psi$ of (28) in a "white $L_{2}-L_{1}$ " prior. Using the same tools as in Appendix B, it can be shown that the general form of a white $L_{2}-L_{1}$ prior under the OI-specific constraints of unit sum and positivity is

$$
J^{\text {prior }}(\boldsymbol{x})=\sum_{p, q} \psi(x(p, q)) / \psi^{\prime}\left(x^{\text {prior }}(p, q)\right)
$$

where $\psi$ is a $L_{2}-L_{1}$ function such as (28), and $\psi^{\prime}$ denotes its first derivative.

A interesting refinement of such priors is to model the observed object with the combination of a correlated $L_{2}$ regularization for the extended component of the object and a white $L_{1}$ regularization for the bright spots [45]. 


\section{AlgORITHMS FOR REGUlarizED IMAGING}

The regularized criterion (23) is not strictly convex and actually often multi-modal, because of missing phase information. Therefore, the solution to the OII problem cannot be simply defined as the minimizer of (23). Actually, it should be defined as the point where the minimization algorithm stops. Most OII algorithms (BSMEM, MIRA, WISARD) are iterative local descent algorithms with the exception of MACIM [10] which uses simulated annealing to search for the object's support. In this section, we present two iterative algorithms designed for OII: MIRA and WISARD. Both are at the state of the art: WISARD ranked second in IBC'04, while MIRA ranked second in IBC'06 and very recently won IBC'08. They are able, like MACIM, to handle various prior terms - while BSMEM is dedicated to entropic regularization. They differ, however, in their treatment of the phase problem. There are essentially two approaches of this problem: explicit algorithms use a set of phase variables and proceed with a joint minimization over these variables and the object $x$, while implicit approaches search for a minimum of (23) with respect to $\boldsymbol{x}$, often with some heuristics in order to avoid getting trapped in local minima. Explicit algorithms include VLBMEM [9], WIPE [46], [47] and WISARD. BSMEM, MACIM and MIRA are implicit algorithms. In this respect, MIRA and WISARD are representative of the two main streams of current OII algorithms.

\section{A. Direct Minimization (MIRA)}

The MIRA [48], [49] method (MIRA stands for Multi-aperture Image Reconstruction Algorithm) seeks for the image by minimizing directly criterion (23). MIRA accounts for power spectrum and closure phase data via penalties defined in (13) and (14). MIRA implicitly accounts for missing phase information, as it only searches for the object $\boldsymbol{x}$. Since MIRA does not attempt to explicitly solve degeneracies, it can be used to restore an image (of course with at least a $180^{\circ}$ orientation ambiguity) from the power spectrum only, i.e., without any phase information, see examples in [49], [50].

To minimize the criterion, the optimization engine is VMLMB [51], a limited memory variable metric algorithm which accounts for parameter bounds. This last feature is used to enforce positivity of the solution. Only the value of the cost function and its gradient are needed by VMLMB. Normalization of the solution is obtained by a change of variables, i.e., the image brightness distribution becomes $x_{k}=z_{k} / \sum_{j} z_{j}$, where $\boldsymbol{z}$ are the internal variables seen by the optimizer with the constraints that $z_{k} \geq 0, \forall k$. Thus, $\boldsymbol{x}$ is both normalized and positive. The gradient is modified as follows:

$$
\begin{aligned}
\partial J / \partial z_{k} & =\sum_{j}\left(\partial J / \partial x_{j}\right)\left(\partial x_{j} / \partial z_{k}\right) \\
& =\left(\partial J / \partial x_{k}-\sum_{j} x_{j} \partial J / \partial x_{j}\right) / \sum_{\ell} z_{\ell} .
\end{aligned}
$$

To avoid getting trapped into a local minimum of the data penalty $J^{\text {data }}$ which is multi-modal, MIRA starts the minimization with a purposely too high regularization level. After convergence, the reconstruction is restarted from the previous solution with a smaller regularization level (e.g. the value of $\mu$ is divided by two). These iterations are repeated until the chosen value of $\mu$ is reached. This procedure mimics the more clever strategy proposed by Skilling \& Bryan [52] and which is implemented in MemSys the optimization engine of BSMEM.

\section{B. A Self Calibration Approach (WISARD)}

The self calibration approach developed in [22], [38], [53] relies on an explicit modeling of the missing phase information and allows one to obtain a convex intermediate image reconstruction criterion. It is inspired by self-calibration algorithms in radio-interferometry [54], but uses a more precise approximation of the observation model than first attempts such as [47].

This approach consists in jointly finding the object $\boldsymbol{x}$ and an $\left(N_{\mathrm{T}}-1\right)$-dimensional phase vector $\boldsymbol{\alpha}$, corresponding to phase components in the closure operator kernel of (9). It starts from a generalized inverse solution to the phase closure equation (12), using the operator $\boldsymbol{C}^{\dagger} \triangleq \boldsymbol{C}^{\mathrm{T}}\left(\boldsymbol{C} \boldsymbol{C}^{\mathrm{T}}\right)^{-1}$. By applying $\boldsymbol{C}^{\dagger}$ on the left to (12) and (9), the missing phase components are made explicit

$$
\exists \boldsymbol{\alpha}(t) \mid \boldsymbol{C}^{\dagger} \boldsymbol{\beta}^{\text {data }}(t)=\boldsymbol{\phi}(\boldsymbol{x}, t)+\overline{\boldsymbol{B}} \boldsymbol{\alpha}(t)+\boldsymbol{C}^{\dagger} \boldsymbol{\beta}^{\text {noise }}(t) .
$$

It is thus tempting to define a pseudo-equation of visibility phase measurement by identifying the term $\boldsymbol{C}^{\dagger} \boldsymbol{\beta}^{\text {noise }}(t)$ of (32) with a noise affecting the visibility phase [55]

$$
\boldsymbol{\phi}^{\text {data }}(t)=\underbrace{\boldsymbol{\phi}(\boldsymbol{x}, t)+\overline{\boldsymbol{B}} \boldsymbol{\alpha}(t)}_{\boldsymbol{\phi}(\boldsymbol{x}, \boldsymbol{\alpha}(t), t)}+\boldsymbol{\phi}^{\text {noise }}(t) .
$$

Unfortunately, as matrix $C^{\dagger}$ is singular, this identification is not rigorously possible and one is led to associate an ad hoc covariance matrix $\boldsymbol{R}_{\phi}$ with the term $\boldsymbol{\phi}^{\text {noise }}(t)$ so as to approximately fit the statistical behavior of the closures. Recently, [22], [38] have discussed possible choices for $\boldsymbol{R}_{\phi}$.

Finding a suitable approximation for the covariance of the amplitude measurements (12), cf. [22] and [38], gives a "myopic" measurement model, i.e., one that depends on the unknowns $\boldsymbol{x}$ and $\boldsymbol{\alpha}$

$$
\left\{\begin{array}{l}
\boldsymbol{a}^{\text {data }}(t)=\boldsymbol{a}(\boldsymbol{x}, t)+\boldsymbol{a}^{\text {noise }}(t) \\
\boldsymbol{\phi}^{\text {data }}(t)=\boldsymbol{\phi}(\boldsymbol{x}, \boldsymbol{\alpha}(t), t)+\boldsymbol{\phi}^{\text {noise }}(t)
\end{array}\right.
$$

with Gaussian noise terms on the modulus and on the amplitude. Still, the resulting likelihood is not quadratic with respect to $\boldsymbol{x}$, because a Gaussian additive noise in phase and modulus is not equivalent to an additive complex Gaussian noise on the visibility. This is the problem of converting measurements from polar coordinates to Cartesian ones, which has long been known in the field of radar [56] and was identified only very recently in optical interferometry [57]. The myopic model of (34) is thus further approximated by a complex additive Gaussian model such as

$$
\boldsymbol{y}^{\mathrm{data}}(t)=\boldsymbol{a}(\boldsymbol{x}, t) e^{i \boldsymbol{\phi}(\boldsymbol{x}, \boldsymbol{\alpha}(t), t)}+\boldsymbol{y}^{\text {noise }}(t)
$$

The mean value and covariance matrix of the additive complex noise term $\boldsymbol{y}^{\text {noise }}(t)$ can be chosen so that the corresponding data likelihood criterion is convex quadratic w.r.t. the complex 

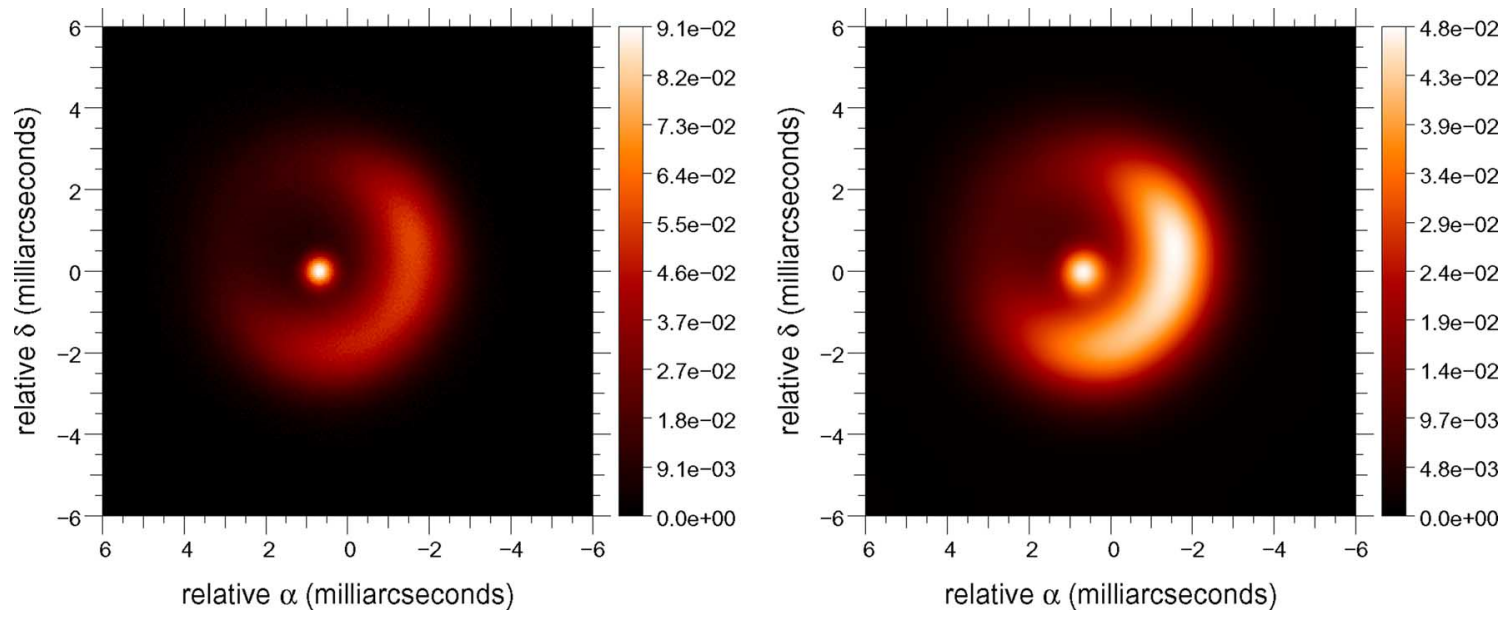

Fig. 2. Synthetic object from IBC'04. Left: original full resolution true object. Right: true object convolved by the PSF of a 132-m perfect telescope.

$\boldsymbol{y}^{\text {model }}(t)=\boldsymbol{a}(\boldsymbol{x}, t) e^{i \boldsymbol{\phi}(\boldsymbol{x}, \boldsymbol{\alpha}(t), t)}$ while remaining close to the real nonconvex likelihood [22], [57]. Finally, using (33)

$$
\boldsymbol{y}^{\text {data }}(t)=(\boldsymbol{H}(t) \boldsymbol{x}) \otimes \exp (i \bar{B} \boldsymbol{\alpha}(t))+\boldsymbol{y}^{\text {noise }}(t)
$$

where $\boldsymbol{H}(t)$ is the discrete time FT matrix corresponding to the instantaneous frequency coverage at time $t$ and $\otimes$ denotes component-wise multiplication of vectors. As clearly shown by (36), the resulting model is now linear in $\boldsymbol{x}$ for a fixed $\boldsymbol{\alpha}$.

This last step leads to a data fitting term $J_{\text {wisard }}^{\text {data }}$ that is quadratic in the real and imaginary parts of the residuals-see [22] and [38] for a complete expression. As discussed in Section III, this data term is then combined with a convex regularization term, so as to obtain a composite criterion

$$
J^{\text {wisard }}(\boldsymbol{x}, \boldsymbol{\alpha})=J_{\text {wisard }}^{\text {data }}(\boldsymbol{x}, \boldsymbol{\alpha})+\mu J^{\text {prior }}(\boldsymbol{x}) .
$$

Let us emphasize the interesting properties of $J^{\text {wisard }}$. On one hand $\boldsymbol{x} \mapsto J^{\text {wisard }}(\boldsymbol{x}, \cdot)$ is convex in $\boldsymbol{x}$; on the other hand, $\boldsymbol{\alpha} \mapsto J^{\text {wisard }}(\cdot, \boldsymbol{\alpha})=J_{\text {wisard }}^{\text {data }}(\cdot, \boldsymbol{\alpha})$ is separable over measurement instants $t$, which allows handling the phase step by several parallel low-dimensional optimizations.

The WISARD algorithm makes use of these properties and minimizes $J^{\text {wisard }}$ alternately in $\boldsymbol{x}$ for the current $\boldsymbol{\alpha}$ and in $\boldsymbol{\alpha}$ for the current $x$. The structure of WISARD is the following: after a first step that casts the true data model into the myopic model (34), a second step "convexifies" the obtained model w.r.t. $\boldsymbol{x}$, to obtain the model of (36). After the selection of the guess and the prior, WISARD performs the alternating minimization.

For the moment, this approach is less versatile than a direct all-purpose minimization method such as MIRA: WISARD cannot cope with missing phase closure information or take into account bispectrum moduli. Indeed, as the pseudo-likelihood associated to model (36) is derived, data that do no fit this recasting stage are not taken into account. Extending WISARD to make it more versatile in the above-mentioned sense deserves some future work.

\section{Results on Synthetic Data}

This section presents results of nonparametric reconstruction methods on the synthetic interferometric data that were pro-

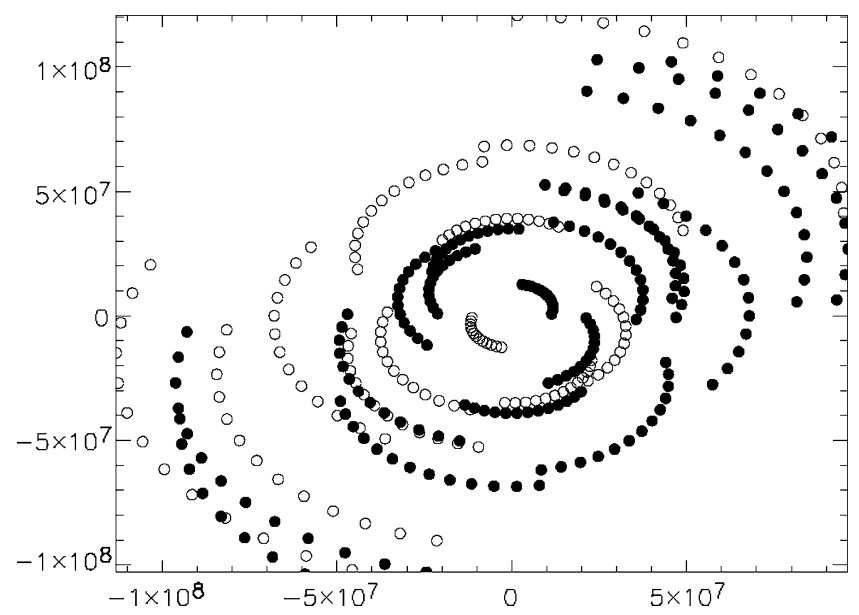

Fig. 3. Frequency coverage from the IBC' 04 .

duced by C. Hummel for the 2004 International Imaging Beauty Contest (IBC'04) organized by P. Lawson for the IAU [9]. These data simulate the observation of the synthetic object shown in Fig. 2 with the NPOI 6-telescope interferometer. The corresponding frequency coverage, shown in Fig. 2, contains 195 square visibility moduli and 130 closure phases. The resolution of the interferometric configuration, as given by the ratio of the minimum wavelength over the maximum baseline, is 0.9 mas.

In Fig. 2 right, we present the image that a $132-\mathrm{m}$ perfect telescope would provide of the object. The cutoff frequency of such an instrument would be twice the maximum value of the frequency coverage used to produce the synthetic dataset (see Fig. 3). It is therefore relevant to compare the reconstructions with this image.

Various results of MIRA with quadratic regularizations are presented in Fig. 4. The top image is essentially a "dirty reconstruction": it uses a separable quadratic penalty with a very low value of regularization parameter $\mu$. The middle image is obtained in the same setting, but with a positivity constraint. The improvement is dramatic, as both the object support and its low resolution features are recovered. An interpretation is that the positivity plays the role of a floating support constraint, which 

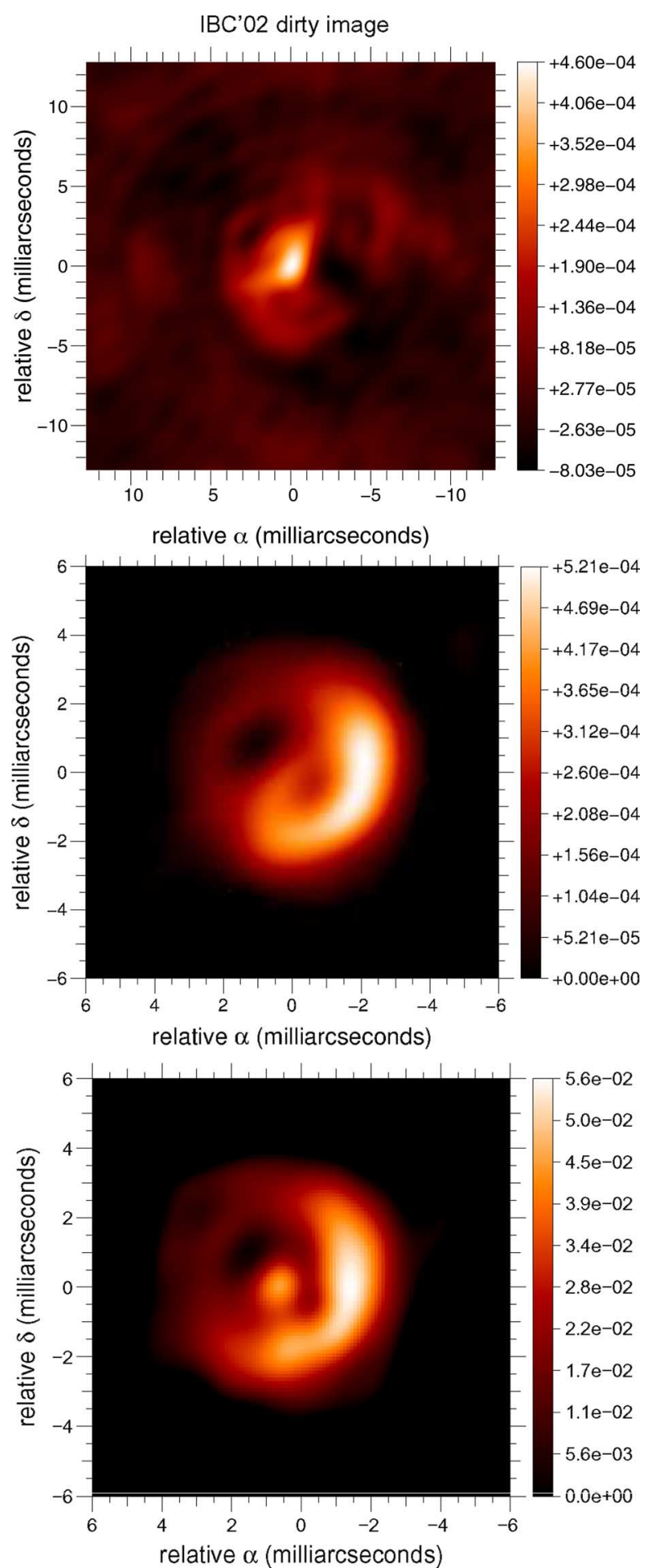

Fig. 4. Results on IBC'04 with Mira. Top: "dirty" reconstruction (see text). Middle: positivity constraint. Bottom: soft support quadratic regularization (25), with prior object Lorentzian of 5 mas FWHM and 0.1 mas pixel size. All reconstructions have $256 \times 256$ pixels.

favors smooth spectra and interpolates the missing spatial frequencies. The bottom image uses the soft support quadratic regularization of (25), with a Lorentzian of 5 mas FWHM as a prior object and a positivity constraint. This regularization, although quadratic, leads to a very good reconstruction, with a central peak clearly separated from the asymmetric shell.
Fig. 5 presents two WISARD reconstructions. The left one is obtained with the same soft support quadratic regularization than the MIRA reconstruction Fig. 4, bottom. Although MIRA and WISARD are based on different criteria and follow different paths during the optimization, the reconstructions are visually very close. With such a "rich" 6-telescope dataset the missing phase information $(33 \%)$ is reasonable and the differences between reconstructions, when they are present, originate mainly from the choice of different regularization terms. As an example, a reconstruction based on the white $L_{2}-L_{1}$ spike-preserving prior of (30) with a constant prior object is shown in Fig. 5, right. This last reconstruction presents finer details than quadratic ones, possibly even finer than the smoothed object of Fig. 2, at the price of some artefacts on the asymmetric shell. However, the validity of these details is difficult to assess.

As a conclusion, the proposed soft support quadratic regularization yields images of quality comparable to those obtained with spike-preserving priors. Contrarily to what is generally believed (see for instance Narayan and Nityananda [43]), special-purpose quadratic separable regularizations are perfectly suitable for image reconstruction by Fourier synthesis as soon as the object is compact and positivity constraints are active.

\section{Processing Real Data}

\section{A. The Infrared Optical Telescope Array (IOTA)}

The IOTA interferometer, operated from 1993 to 2006 (cf., tdc-www.harvard.edu/iota/) on Mt Hopkins (Arizona, USA), had variable baseline lengths and thus gave access to a broad frequency coverage. It operated with three $45 \mathrm{~cm}$ siderostats that could be located at different stations on each arm of an L-shaped array (the NE arm is $35 \mathrm{~m}$ long, the SE arm $15 \mathrm{~m}$ ). The maximum nonprojected baseline length was $38 \mathrm{~m}$, and the minimum one $5 \mathrm{~m}$. It used fiber optics for spatial filtering, and an integrated optics beam combiner called IONIC [58]. It was decommissioned in July 2006.

\section{B. The Dataset}

The dataset presented here correspond to observations of the star $\chi$ Cygni. Of the class of the Mira variables, $\chi$ Cygni is an evolved star whose extended atmosphere is puffed up by the strong radiation pressure induced by fusion of metals (here, metals means chemical elements heavier than Helium) in its core. This late stage of evolution is appropriate for interferometric imaging since the large stellar radius can be resolved by optical interferometers. Moreover, these stars are usually bright in the infrared, allowing robust fringe tracking.

$\chi$ Cygni was observed during a six-night run in May 2006. Night-time is used for observing and daytime is used to move and configure the telescopes. The log of the interferometer configurations is presented in Table I.

The reduced dataset is plotted in the two panels of Fig. 6. $\chi$ Cygni was observed over the whole $\mathrm{H}$ Band $(1.5 \mu \mathrm{m}<\lambda<$ $1.8 \mu \mathrm{m})$, and fringes were dispersed in order to obtain spectral information. In this paper, we shall not address the chromaticity of the object. Therefore, we use the diverse wavelengths only as a way to increase the Fourier coverage. The frequency plane coverage was previously presented in Fig. 1 . The visibilities are 

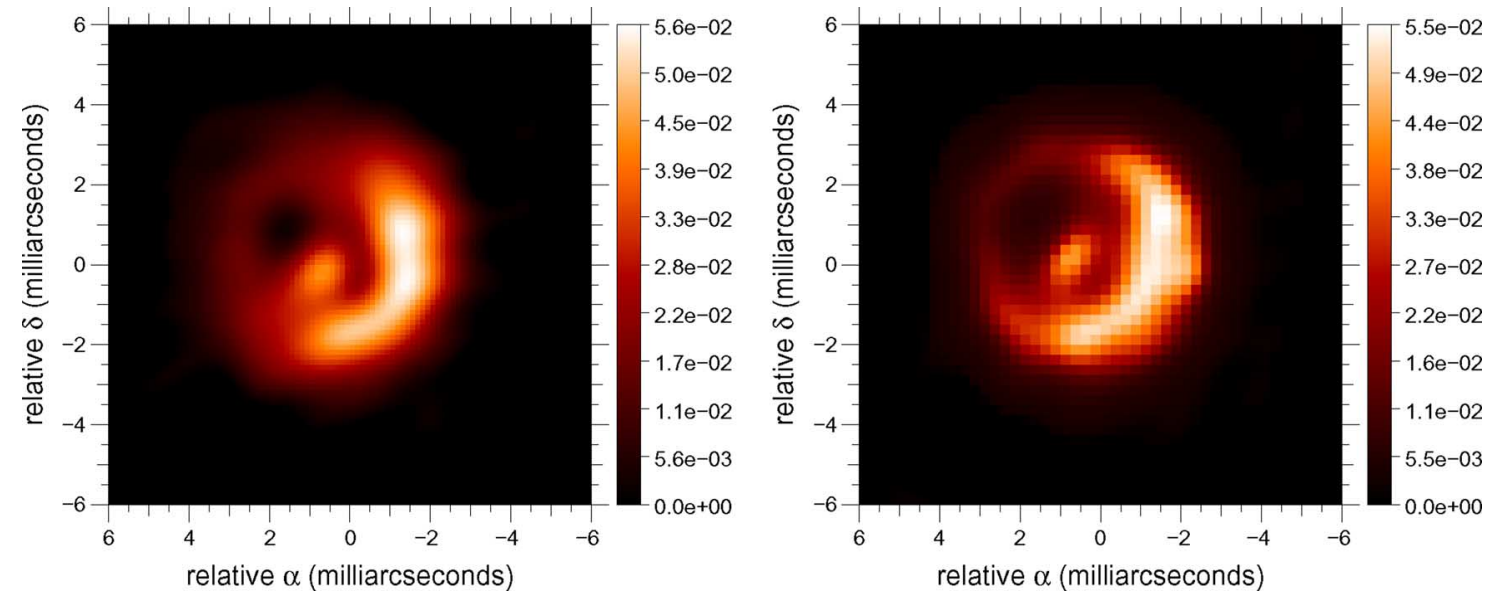

Fig. 5. Results on IBC'04 with Wisard. Left: $120 \times 120$ pixels reconstruction with 0.125 mas pixel size using a soft support quadratic regularization (25), with prior object Lorentzian of 2.5 mas FWHM. Right: $60 \times 60$ pixels reconstruction with 0.25 mas pixel size based on a white $L_{2}-L_{1}$ regularization (30) with a constant prior object and parameters $\delta=1, s=1 / 2 P Q$.

TABLE I

$\chi$ CYGNI OBSERVING LOG

\begin{tabular}{crrr}
\hline \hline Date & \multicolumn{3}{c}{ Configuration $^{\mathrm{a}}$} \\
(UT) & A & B & C \\
\hline 2006 May 11 & 15 & 5 & 10 \\
2006 May 12 & 15 & 5 & 0 \\
2006 May 13 & 15 & 15 & 0 \\
\hline
\end{tabular}

\begin{tabular}{crrr}
\hline \hline Date & \multicolumn{3}{c}{ Configuration } \\
(UT) & A & B & C \\
\hline 2006 May 14 & 30 & 15 & 0 \\
2006 May 15 & 35 & 15 & 21 \\
2006 May 16 & 35 & 15 & 25 \\
\hline
\end{tabular}

a Configuration refers to the location in meters of telescopes $\mathrm{A}, \mathrm{B}, \mathrm{C}$ on the NE, SE, and NE arms, respectively. Position "0" corresponds to the arms' intersection.

presented in the upper panel of Fig. 6 as a function of the baseline length in wavelength units. The closure phases are plotted on the bottom panel. Due to the difficulty to represent these phases as a function of a physical parameter, we simply present them as a function of the observation data-point number. The vertical lines indicate a change of interferometer configuration.

A closure phase equals to zero or $\pi$ corresponds to a centrosymmetric object. Thus, a preliminary inspection of the closure phases can show the presence of asymmetries. The higher the frequency, the more apparent the asymmetry is. This makes sense to an astronomer because photospheric inhomogeneities are likely to be present at a smaller scale than the size of the photosphere. In the case of $\chi$ Cygni, the photosphere's size estimate is 21.3 mas, to be compared with the resolution of the interferometer, slightly less than 5 mas.

\section{Image Reconstruction}

In Fig. 7, we present three reconstructed images, obtained using different methods and priors.

The first image corresponds to a parametric inversion of the data using all the available spectral channels merged together. The justification for such a polychromatic processing of the data is ongoing work, however first results confirm a variation of the angular diameter of less than 1 mas in the $\mathrm{H}$ band (S. Lacour, private communication, 2008).

As stated, the quality of the reconstruction will depend heavily on the correctness of the model of the object. Fortunately, Mira variables are not completely unknown, and
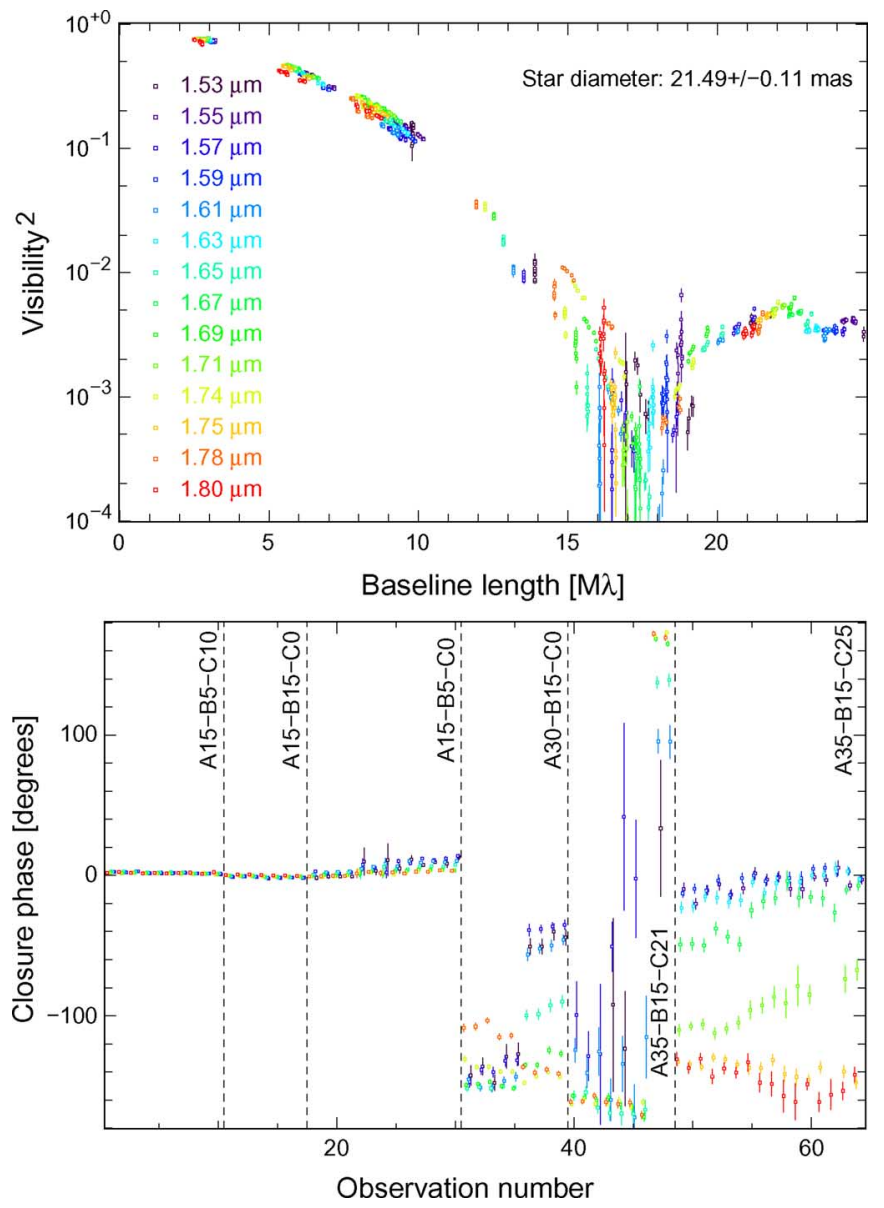

Fig. 6. IOTA dataset on $\chi$ Cyg. Top panel: Visibility square $\boldsymbol{s}^{\text {data }}$ as a function of the baseline length. Bottom panel: closures phases $\boldsymbol{\beta}^{\text {data }}$ as a function of the observation number. Labels of the type Axx-Bxx-Cxx correspond to the telescope configurations (see Table I).

previous astronomical observations tell us that the star is expected to possess: i) a large limb-darkened photosphere; ii) important asymmetries of the form of photospheric "hot-spots"; and iii) a close, warm, molecular layer surrounding the photosphere at around one stellar radius of the photosphere. 


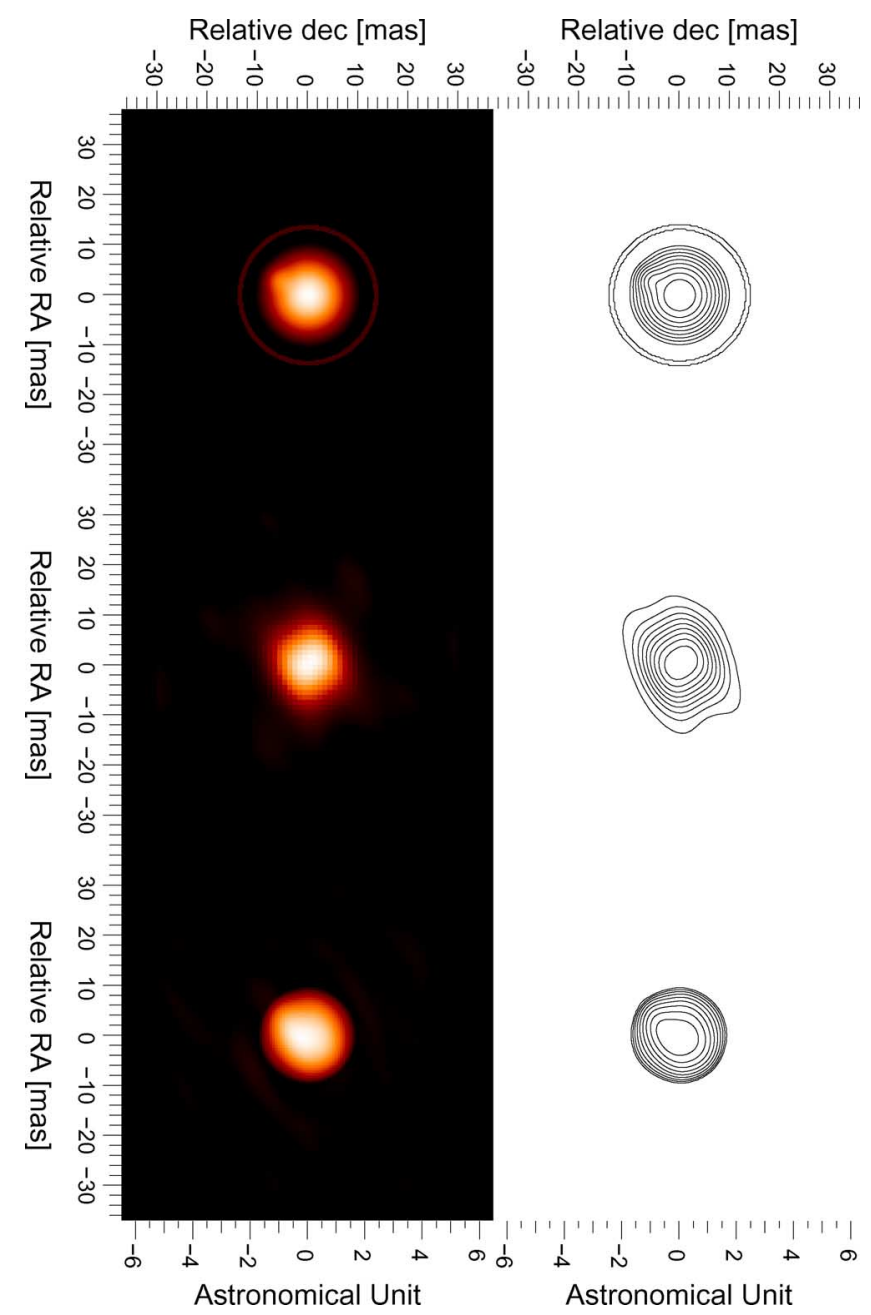

Fig. 7. Reconstructed images of the star $\chi$ Cygni, right: contour plot with levels $10 \%, 20 \%, \ldots, 90 \%$ of the maximum. From top to bottom: parametric reconstruction, WISARD with white $L_{2}-L_{1}$ prior, MIRA with a separable quadratic penalization towards a prior parametric solution. Details on the different methods are given in Section V-C. Note the colossal size of the star: at the distance of $\chi$ Cygni (170 pc; [59]), 5 mas correspond roughly to the distance Sun-Earth (1 astronomical unit).

This simplistic theoretical model (i.e., a limb darkened disk, a spot and a spherical thin layer) is converted to a geometrical parametric model, which is adjusted to the data through the minimization of $J^{\text {data }}(x(\boldsymbol{\theta}))$ of (13). The image presented in the left panel of Fig. 7 corresponds to the geometrical model with best fit parameters.

These parameters give direct information on the structure of the object, and error bars can be estimated: the star diameter is $21.49 \pm 0.11$ mas and the hotspot contrast is $1.70 \pm 0.04 \%$. Note that the requirements of a parametric reconstruction, in terms of frequency coverage, are much less stringent than that of a nonparametric one. Thus, parametric inversion can also be used with each spectral channel separately, to determine the spectral energy distribution of the surrounding atmospheric layer.

The second image was produced using the WISARD software described in Section IV-B with a white $L_{2}-L_{1}$ prior-see (26). The last image was reconstructed using MIRA, see Section IV-A, and more importantly, using a prior solution in the white quadratic setting of (25). The prior solution is a limb-darkened disk whose parameters are determined by model fitting on the visibilities.

\section{Discussion}

Fig. 7 shows that, for the sparse data at hand, the more stringent the prior, the more convincing the reconstruction looks to an astronomer.

More precisely, the white $L_{2}-L_{1}$ prior used by WISARD does not allow to distinguish more than a resolved photosphere and the fact that some asymmetry is present. The form of the reconstructed photosphere and its surrounding can be questioned when compared to what is expected from the theory. Besides, on the presented reconstructions, MIRA was used with a much more informative prior and is in good agreement with the parametric reconstruction. This image is however interesting because the reconstruction is notably different from a simple disk, and adds an asymmetry - a "hotspot" —on the surface. The presence of an asymmetry could be foreseen by looking at the raw closure phases (right panel of Fig. 6). The fact that this asymmetry appears similarly — in terms of flux and position-on the parametric and on the nonparametric image reconstructions is a convincing argument to validate both images.

Note that, on the MIRA reconstruction, an emission surrounding the photosphere is present, but its reality is difficult to assert on the reconstructed image. Hence, it should be pointed out that neither of the nonparametric reconstructions exhibits the molecular layer which is revealed by the parametric reconstruction.

\section{CONCLUSIONS}

In recent years, long baseline optical interferometers with better capabilities have become available. Routine observations with three or more telescope interferometers have become a reality. Although quite sparse with respect to radio arrays, the spatial frequency coverage allows one to study more complex objects and to reconstruct images. In this paper, we have described, besides the parametric reconstruction approach, two nonparametric image reconstruction methods, MIRA and WISARD. MIRA is based on the direct optimization of a Bayesian criterion while WISARD adopts a self-calibration approach inspired from radioastronomy. As such, these two methods are representative of the two families of state-of-the-art nonparametric reconstruction methods [9], [10].

On rich-enough data, which are currently available only from simulations, both methods demonstrate a valuable and comparable capability for imaging complex objects. On such data, the differences between reconstructions originate mostly from the choice of different regularization terms. We have reviewed common regularization criteria and proposed an original regularization criterion that can be used on compact objects to enforce a "soft support" constraint. This criterion, although it is quadratic, yields images of quality comparable to that obtained with spike-preserving priors on the IBC'04 dataset.

We have demonstrated the operational imaging capabilities of these methods on a IOTA dataset of $\chi$ Cygni. However, for these data, which may be considered representative of today's optical long-baseline interferometers, we have shown that the parametric approach remains a choice of reference for OII. 
The experience gathered while trying to extract the most information from real-world data, both in the work described here and elsewhere [31], suggests that the optimal processing of measurements from present-day interferometers should make use of both approaches in an alternate fashion as described below. With a sparse frequency coverage, a parametric reconstruction is useful to obtain $a b$ initio a first estimate for the observed object. A parametric reconstruction will not reveal any unguessed feature, but it can be used to guide nonparametric reconstructions as an initial guess or as a prior object for instance. Then the reconstructed images are very useful to understand the structure of a complex object since they are most often the very first insight one gets about the source at this angular resolution.

The fidelity of nonparametric reconstructions remains limited in a photometric sense and can therefore seldom be used to infer astrophysical parameters. In fine, parametric models remain the choice of reference for estimating astrophysical parameters related to the very physics of the objects of interest.

It is therefore very likely that even in the yet to come imaging era of optical interferometry, i.e., when much larger optical interferometric arrays become operational, the parametric approach will remain a useful tool for astrophysical modeling, even though it will no longer be necessary to initialize the imaging process.

\section{APPENDIX A}

\section{The Closure and Baseline Operators $\boldsymbol{C}$ and $\boldsymbol{B}$}

Let $N_{\mathrm{T}}$ be the number of telescopes of a complete interferometric array. We have the following definitions:

$$
\begin{aligned}
& \boldsymbol{B}_{2} \triangleq\left[\begin{array}{ll}
-1 & 1
\end{array}\right], \boldsymbol{B}_{N_{\mathrm{T}}} \triangleq\left[\begin{array}{c|c}
-\mathbf{1}_{N_{\mathrm{T}}-1} & \mathbf{I d}_{N_{\mathrm{T}}-1} \\
\hline \boldsymbol{O} & \boldsymbol{B}_{N_{\mathrm{T}}-1}
\end{array}\right] \\
& \boldsymbol{C}_{N_{\mathrm{T}}} \triangleq\left[-\boldsymbol{B}_{N_{\mathrm{T}}-1} \mid \mathbf{I}_{\frac{\left(N_{\mathrm{T}}-1\right)\left(N_{\mathrm{T}}-2\right)}{2}}\right]
\end{aligned}
$$

for $N_{\mathrm{T}} \geq 3$. It is easy to see that $\boldsymbol{C B}=\mathbf{0}$. The generalized inverse $\boldsymbol{C}^{\dagger}$ of $\boldsymbol{C}$, defined by $\boldsymbol{C}^{\dagger} \triangleq \boldsymbol{C}^{\mathrm{T}}\left[\boldsymbol{C C}^{\mathrm{T}}\right]^{-1}$, is such that $\boldsymbol{C} \boldsymbol{C}^{\dagger}=\boldsymbol{I} \mathrm{d}$.

\section{APPENDIX B \\ QUADRATIC REGULARIZATION TOWARDS A PRIOR OBJeCt IN OI}

A general expression for a quadratic separable regularization is given by

$$
J^{\text {prior }}(\boldsymbol{x})=\sum_{p, q} w(p, q) x(p, q)^{2}
$$

where $w(p, q)>0, \forall p, q$, otherwise the criterion is degenerated. The default solution $\boldsymbol{x}^{\text {prior }}$ is obtained by minimizing the cost function in the absence of data and subject to the constraints (normalization and nonnegativity)

$$
\left\{\begin{array}{l}
\boldsymbol{x}^{\text {prior }}=\arg \min _{\boldsymbol{x}} J^{\text {prior }}(\boldsymbol{x}) \\
\text { s.t. } \quad \boldsymbol{x} \geq 0 \text { and } \sum_{p, q} x(p, q)=1
\end{array}\right.
$$

where $\boldsymbol{x} \geq 0$ means $x(p, q) \geq 0, \forall p, q$. Assuming for the moment that all inequality constraints are inactive at the solution, the Lagrangian for the constrained problem can be written as

$$
\mathcal{L}(\boldsymbol{x} ; \lambda)=J^{\text {prior }}(\boldsymbol{x})-2 \lambda\left(\sum_{p, q} x(p, q)-1\right),
$$

Minimizing $\mathcal{L}(\boldsymbol{x} ; \lambda)$ with respect to $\boldsymbol{x}$ only readily yields $\boldsymbol{x}^{+}(\lambda)=\arg \min _{\boldsymbol{x}} \mathcal{L}(\boldsymbol{x} ; \lambda)=\lambda / \boldsymbol{w}$. The optimal Lagrange multiplier $\lambda^{+}$is identified by requiring the normalization of $\boldsymbol{x}^{+}\left(\lambda^{+}\right)$and, finally, the default solution is

$$
x^{\text {prior }}(p, q)=\left[\boldsymbol{x}^{+}\left(\lambda^{+}\right)\right](p, q)=\frac{1 / w(p, q)}{\sum_{p^{\prime}, q^{\prime}} 1 / w\left(p^{\prime}, q^{\prime}\right)}
$$

which is normalized and strictly positive since $\boldsymbol{w}>0$. This additionally validates our hypothesis that the inequality constraints were all inactive at the solution. Combining (39) and (42) yields the expression of the quadratic, separable, loose support regularization term of (25).

\section{ACKNOWLEDGMENT}

The authors would like to thank all the people who contributed to the existence and success of the IOTA interferometer. They also thank the anonymous reviewers for their numerous suggestions, which resulted in a great improvement of the paper's quality.

\section{REFERENCES}

[1] A. Quirrenbach, "Optical interferometry," Annu. Rev. Astron. and Astrophys., 2001.

[2] J. D. Monnier, "Optical interferometry in astronomy," Rep. Progr. Phys., vol. 66, pp. 789-857, May 2003.

[3] J. D. Monnier et al., "Imaging the surface of Altair," Science, vol. 317, p. 342, Jul. 2007.

[4] A. D. da Souza et al., "The spinning-top be star achernar from VLTIVINCI," Astron. Astrophys., vol. 407, no. 3, pp. L47-L50, 2003.

[5] W. Jaffe et al., "The central dusty torus in the active nucleus of NGC 1068," Nature, vol. 429, pp. 47-49, 2004.

[6] A. Poncelet, G. Perrin, and H. Sol, "A new analysis of the nucleus of NGC 1068 with MIDI observations," Astron. Astrophys., vol. 450, pp. 483-494, 2006.

[7] K. R. W. Tristram et al., "Resolving the complex structure of the dust torus in the active nucleus of the circinus galaxy," Astron. Astrophys., vol. 474, pp. 837-850, 2007.

[8] P. R. Lawson, "Notes on the history of stellar interferometry," in Principles of Long Baseline Stellar Interferometry, Course Notes from 1999 Michelson Summer School, P. R. Lawson, Ed. Pasadena, CA: NASAJPL, 2000, pp. 325-32, no. 00-009.

[9] P. R. Lawson et al., "An interferometric imaging beauty contest," in New Frontiers in Stellar Interferometry, Proc. SPIE Conf., W. A. Traub, Ed. Bellingham, WA: SPIE, 2004, vol. 5491, pp. 886-899.

[10] P. R. Lawson et al., "The 2006 interferometry imaging beauty contest," in Advances in Stellar Interferometry, J. D. Monnier, M. SchÖller, and W. C. Danchi, Eds. Bellingham, WA: SPIE, 2006, vol. 6268, p. 59.

[11] J. W. Goodman, Statistical Optics. New York: Wiley, 1985.

[12] M. Born and E. Wolf, Principles of Optics, 6th ed. New York: Pergamon, 1993.

[13] J.-M. Mariotti, "Introduction to Fourier optics and coherence," in Diffraction-Limited Imaging With Very Large Telescopes, ser. NATO ASI Series C, D. M. Alloin and J.-M. Mariotti, Eds. Norwell, MA: Kluwer, 1989, vol. 274, pp. 3-31.

[14] F. Roddier, "The effects of atmospherical turbulence in optical astronomy," in Progress in Optics, E. Wolf, Ed. Amsterdam, The Netherlands: North Holland, 1981, vol. XIX, pp. 281-376. 
[15] F. Roddier, J. M. Gilli, and G. Lund, "On the origin of speckle boiling and its effects in stellar speckle interferometry," J. Opt., vol. 13, no. 5, pp. 263-271, 1982.

[16] J.-M. Conan, G. Rousset, and P.-Y. Madec, "Wave-front temporal spectra in high-resolution imaging through turbulence," J. Opt. Soc. Amer. A, vol. 12, no. 12, pp. 1559-157, Jul. 1995.

[17] D. L. Fried, "Statistics of a geometric representation of wavefront Distortion," J. Opt. Soc. Amer., vol. 55, no. 11, pp. 1427-143, 1965.

[18] F. Roddier, Ed., Adaptive Optics in Astronomy. Cambridge, U.K.: Cambridge Univ. Press, 1999.

[19] D. Mourard, I. Bosc, A. Labeyrie, L. Koechlin, and S. Saha, "The rotating envelope of the hot star Gamma Cassiopeiae resolved by optical interferometry," Nature, vol. 342, pp. 520-522, Nov. 1989.

[20] W. J. Tango and R. Q. Twiss, "Michelson stellar interferometry," in Progr. Opt. (A81-13109 03-74) . Amsterdam, The Netherlands: North-Holland Publishing, 1980, vol. 17 , pp. 239-277.

[21] R. C. Jennison, "A phase sensitive interferometer technique for the measurement of the fourier transforms of spatial brightness distribution of small angular extent," Monthly Notices Roy. Astron. Soc., vol. 118, pp. 276-284, 1958.

[22] S. Meimon, L. M. Mugnier, and G. Le Besnerais, "A self-calibration approach for optical long baseline interferometry," J. Opt. Soc. Amer. $A$, accepted for publication.

[23] J. D. Monnier et al., "First results with the IOTA3 imaging interferometer: The spectrocopic binaries $\lambda$ virginis and WR 140," apJL, vol. 602, no. 1, pp. L57-L60, Feb. 2004.

[24] J. C. Dainty and A. H. Greenaway, "Estimation of spatial power spectra in speckle interferometry," J. Opt. Soc. Amer., vol. 69, no. 5, pp. 786-79, May 1979.

[25] B. Wirnitzer, "Bispectral analysis at low light levels and astronomical speckle masking," J. Opt. Soc. Amer. A, vol. 2, no. 1, pp. 14-2, Jan. 1985.

[26] T. Pauls et al., "A data exchange standard for optical (visible/ir) interferometry," in New Frontiers in Stellar Interferometry, Proc. SPIE Conf. . Bellingham, WA: SPIE, 2004, vol. 5491.

[27] G. Perrin, "The calibration of interferometric visibilities obtained with single-mode optical interferometers. Computation of error bars and correlations," Advanc. Appl. Prob., vol. 400, pp. 1173-1181, Mar. 2003.

[28] R. T. Rockafellar, Convex Analysis. Princeton, NJ: Princeton Univ. Press, 1996.

[29] D. Hestroffer, "Centre to limb darkening of stars. New model and application to stellar interferometry," A\&A, vol. 327, pp. 199-206, Nov. 1997.

[30] A. Manduca, R. A. Bell, and B. Gustafsson, "Limb darkening coefficients for late-type giant model atmospheres," $A \& A$, vol. 61, pp. 809-813, Dec. 1977.

[31] S. Lacour et al., "The limb-darkened Arcturus; Imaging with the IOTA/ IONIC interferometer," ArXiv E-Prints, vol. 804, Apr. 2008.

[32] G. Perrin et al., "Study of molecular layers in the atmosphere of the supergiant star $\mu$ Cep by interferometry in the K band," $A \& A$, vol. 436 , pp. 317-324, Jun. 2005.

[33] F. R. Schwab, "Optimal gridding of visibility data in radio interferometry," in Measurement and Processing for Indirect Imaging, J. A. Roberts, Ed. Cambridge, U.K.: Cambridge Univ. Press, 1984, p. 333.

[34] A. Lannes, E. Anterrieu, and K. Bouyoucef, "Fourier interpolation and reconstruction via Shannon-type techniques. I regularization principle," J. Mod. Opt., vol. 41, no. 8, pp. 1537-1574, 1994.

[35] A. Lannes, S. Roques, and Casanove, "Stabilized reconstruction in signal and image processing: Part i: Partial deconvolution and spectral extrapolation with limited field," J. Mod. Opt., vol. 34, pp. 161-226, 1987.

[36] A. Lannes, E. Anterrieu, and P. Maréchal, "Clean and wipe," Astron. and Astrophys. Suppl., vol. 123, pp. 183-198, May 1997.

[37] J.-M. Conan, L. M. Mugnier, T. Fusco, V. Michau, and G. Rousset, "Myopic deconvolution of adaptive optics images using object and point spread function power spectra," Appl. Opt., vol. 37, no. 21, pp. 4614-4622, Jul. 1998.

[38] S. Meimon, "Reconstruction d'images astronomiques en interférométrie optique," Ph.D. dissertation, Université Paris Sud, Paris, France, 2005.

[39] A. Blanc, L. M. Mugnier, and J. Idier, "Marginal estimation of aberrations and image restoration by use of phase diversity," J. Opt. Soc. Amer. A, vol. 20, no. 6, pp. 1035-1045, 2003.
[40] L. M. Mugnier, C. Robert, J.-M. Conan, V. Michau, and S. Salem, "Myopic deconvolution from wavefront sensing," J. Opt. Soc. Amer. A, vol. 18, pp. 862-872, Apr. 2001.

[41] W. J. Rey, Introduction to Robust and Quasi-Robust Statistical Methods. Berlin, Germany: Springer-Verlag, 1983.

[42] S. Brette and J. Idier, "Optimized single site update algorithms for image deblurring," in Proc. IEEE ICIP, Lausanne, Switzerland, Sep. 1996, pp. 65-68.

[43] R. Narayan and R. Nityananda, "Maximum entropy image restoration in astronomy," Ann. Rev. Astron. Astrophys., vol. 24, pp. 127-170, Sep. 1986.

[44] D. F. Buscher, "Direct maximum-entropy image reconstruction from the bispectrum," in IAU Symp. 158: Very High Angular Resolution Imaging, J. G. Robertson and W. J. Tango, Eds., 1994, p. 91, -+.

[45] J.-F. Giovannelli and A. Coulais, "Positive deconvolution for superimposed extended source and point sources," Astron. Astrophys., vol. 439, pp. 401-412, 2005.

[46] L. Delage, F. Reynaud, E. Thiebaut, K. Bouyoucef, P. Marechal, and A. Lannes, "Présentation d'un démonstrateur de synthèse d'ouverture utilisant des liaisons par fibres optiques," in Actes du $16^{\mathrm{e}}$ colloque GRETSI, Grenoble, France, Sep. 1997, pp. 829-832.

[47] A. Lannes, "Weak-phase imaging in optical interferometry," J. Opt. Soc. Amer. A, vol. 15, no. 4, pp. 811-82, Apr. 1998.

[48] E. Thiébaut, P. J. V. Garcia, and R. Foy, "Imaging with Amber/VLTI: The case of microjets," Astrophys. Space. Sci., vol. 286, pp. 171-176, 2003.

[49] E. Thiébaut, "Mira: An effective imaging algorithm for optical interferometry," presented at the Astronomical Telescopes and Instrumentation SPIE Conf., 2008, paper no. 7013-53, vol. 7013.

[50] E. Thiébaut, "Reconstruction d'image en interférométrie optique," in XXIe Colloque GRETSI, Traitement du signal et des images, Troyes, France, 2007.

[51] E. Thiébaut, "Optimization issues in blind deconvolution algorithms," in Astronomical Data Analysis II, Proc. SPIE Conf., J.-L. Starck and F. D. Murtagh, Eds. Bellingham, WA: SPIE, 2002, vol. 4847, pp. 174-183.

[52] J. Skilling and R. K. Bryan, "Maximum entropy image reconstruction: General algorithm," Monthly Not. Roy. Astron. Soc., vol. 211, pp. 111-124, 1984

[53] S. Meimon, L. M. Mugnier, and G. Le Besnerais, "Reconstruction method for weak-phase optical interferometry," Opt. Lett., vol. 30, no. 14, pp. 1809-1811, Jul. 2005.

[54] T. J. Cornwell and P. N. Wilkinson, "A new method for making maps with unstable radio interferometers," Monthly Notices Roy. Astron. Soc., vol. 196, pp. 1067-1086, 1981

[55] A. Lannes, "Integer ambiguity resolution in phase closure imaging," $J$. Opt. Soc. Amer. J. A, vol. 18, pp. 1046-1055, May 2001.

[56] Y. Bar-Shalom and X.-R. Li, Multitarget-Multisensor Tracking: Principles and Techniques. Storrs, CT: Univ. Connecticut, 1995.

[57] S. Meimon, L. M. Mugnier, and G. Le Besnerais, "A convex approximation of the likelihood in optical interferometry," J. Opt. Soc. Amer. A, Nov. 2005.

[58] J.-P. Berger et al., "An integrated-optics 3-way beam combiner for IOTA," in Interferometry for Optical Astronomy II-Proc. SPIE, W. A. Traub, Ed., Feb. 2003, vol. 4838, pp. 1099-1106.

[59] F. Van Leeuwen, Hipparcos, The New Reduction of the Raw Data. New York: Springer, 2007.



Guy Le Besnerais was born in Paris, France, in 1967. He graduated from the École Nationale Supérieure de Techniques Avancées in 1989 and received the Ph.D. degree in physics from the Université de Paris-Sud, Orsay, France, in 1993.

Since 1994, he has been with the Office National d'Études et Recherches Aérospatiales, Châtillon, France. His main interests are in the fields of image reconstruction and spatio-temporal processing of image sequences. He made various contributions in optical interferometry, super-resolution, optical-flow estimation, and 3-D reconstruction from aerial image sequence. 


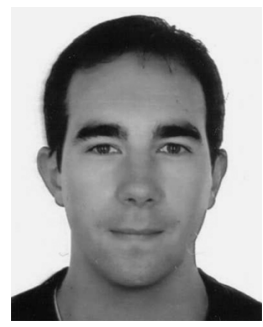

Sylvestre Lacour received the Ph.D. degree in astrophysics in 2007, specializing in astronomical instrumentation, mainly involving interferometry and high angular resolution.

$\mathrm{He}$ is a tenured Assistant Researcher at CNRS $\mathrm{He}$ is working within the National Institute for Earth Sciences and Astronomy, Meudon, France. His astrophysical interests involve the interstellar medium, evolved stars, and extrasolar planets.

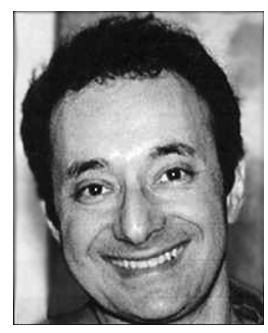

Laurent M. Mugnier graduated from Ecole Polytechnique, France, in 1988. He received the Ph.D. degree in 1992 from Ecole Nationale Supérieure des Télécommunications (ENST), France, for his work on the digital reconstruction of incoherent-light holograms.

In 1994 he joined ONERA, where he is currently a Senior Research Scientist in the field of inverse problems and high-resolution optical imaging. His current research interests include image reconstruction and wavefront-sensing, in particular for adaptive-optics corrected imaging through turbulence, for retinal imaging, for Earth observation and for optical interferometry in astronomy. His publications include five contributions to reference books and 30 papers in peer-reviewed international journals.



Eric Thiébaut was born in Béziers, France, in 1966. He graduated from the École Normale Supérieure in 1987 and received the Ph.D. degree in astrophysics from the Université Pierre and Marie Curie de Paris VII, Paris, France, in 1994.

Since 1995, he has been an Astronomer at the Centre de Recherche Astrophysique de Lyon, France. His main interests are in the fields of signal processing and image reconstruction. He has made various contributions in blind deconvolution, optical interferometry, and optimal detection.

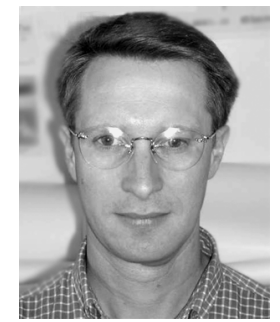

Guy Perrin was born in Saint-Etienne, France, in 1968. He graduated from École Polytechnique in 1992 and received the Ph.D. degree in astrophysics from Université Paris Diderot in 1996.

He has been an Astronomer with Observatoire de Paris since 1999. His research topics focus both on instrumental techniques for high angular resolution observations and on the use of interferometers and telescopes equipped with adaptive optics to study pointlike objects such as evolved stars, active galactic nuclei, and the Galactic Center.

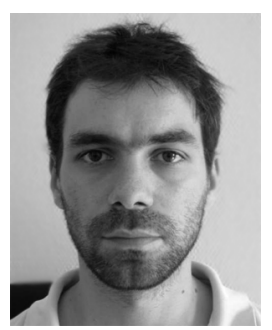

Serge Meimon was born in Paris, France, in 1978. He graduated from École Centrale de Nantes in 2002 and received the Ph.D. degree in physics from Université Paris-Sud Orsay in 2005 for his work on image-reconstruction methods in optical interferometry.

Since then, he has been with the Office National d'Études et Recherches Aérospatiales, Châtillon, France. He has made various contributions in the field of optical high-angular resolution. 